ESAIM: PROCEEDINGS, November 2002, Vol.12, 25-30

M.Thiriet, Editor

\title{
TOWARDS A FIBRE-BASED CONSTITUTIVE LAW FOR THE MYOCARDIUM
}

\author{
Denis Caillerie ${ }^{1}$, Ayman Mourad ${ }^{1,2}$ and Annie Raoult ${ }^{2,3}$
}

\begin{abstract}
According to Streeter, myocardial fibres are organized in a nested set of layers and they are geodesics of these toroidal surfaces. In a previous work, using experimental data, we checked several consequences of this conjecture for the particular case of the left ventricle. We used a fibre reconstruction algorithm that we have now generalized in a surface reconstruction algorithm which can be used for both ventricles and which leads to a better visualization of the layers. In the same time, we are interested in the derivation of a constitutive law for the myocardium which should take into account both the fibre structure and the electric activation. In a first step in this direction, we use a discrete homogenization technique.
\end{abstract}

Résumé. Les fibres myocardiques sont, selon Streeter, des géodésiques de surfaces toroïdales emboîtées. Partant de données expérimentales, nous avons, dans une première étape, vérifié sur le ventricule gauche plusieurs conséquences de cette conjecture. Nous avons maintenant développé un algorithme de reconstruction de surfaces qui permet une visualisation des couches de fibres. Par ailleurs, nous proposons d'utiliser une technique d'homogénéisation discrète pour établir une loi de comportement du muscle cardiaque prenant en compte la structure fibreuse.

AMS subject classification: 74L15, 74Q05, 74Q15, 92B05.

\section{INTRODUCTION}

Knowledge of the geometrical organization of myocardial fibres is a basic requirement for understanding the mechanical design of normal hearts. Dissection techniques are not precise enough for this specific topic since apparently preferred directions are induced by the dissection direction. A new technique was then developed by [Jouk et al., 1995] and [Jouk et al., 2000]. This technique uses quantitative polarized light microscopy devices to provide pointwise measures of the fibres orientation. Data are collected on fetal hearts.

We were first interested in using these data to investigate a conjecture by Streeter according to which myocardial fibres are organized in a nested set of surfaces and, more precisely, are geodesic curves of these surfaces. We explain our method and give our results, all of which back up Streeter's hypothesis, in Section 1. Let us mention that in the particular case of the left ventricle, we use the Clairaut property which is valid for structures of revolution, [Mourad et al., 2001]. In the present paper, using a fibre reconstruction algorithm, we visualize the isovalue surfaces of the Clairaut number. The obtained surfaces clearly show the toroidal structure of the left ventricle wall and the helicoidal pattern of the fibres.

\footnotetext{
1 Laboratoire Sols, Solides, Structures, BP 53, 38041 Grenoble Cedex 9, France; e-mail: Denis.Caillerie@inpg.fr

${ }^{2}$ Laboratoire de Modélisation et Calcul, BP 53, 38041 Grenoble Cedex 9, France; e-mail: Ayman.Mourad@imag.fr

${ }^{3}$ Laboratoire TIMC, Domaine de la Merci, 38076 La Tronche Cedex, France; e-mail: Annie.Raoult@imag.fr
}

(c) EDP Sciences, SMAI 2003 

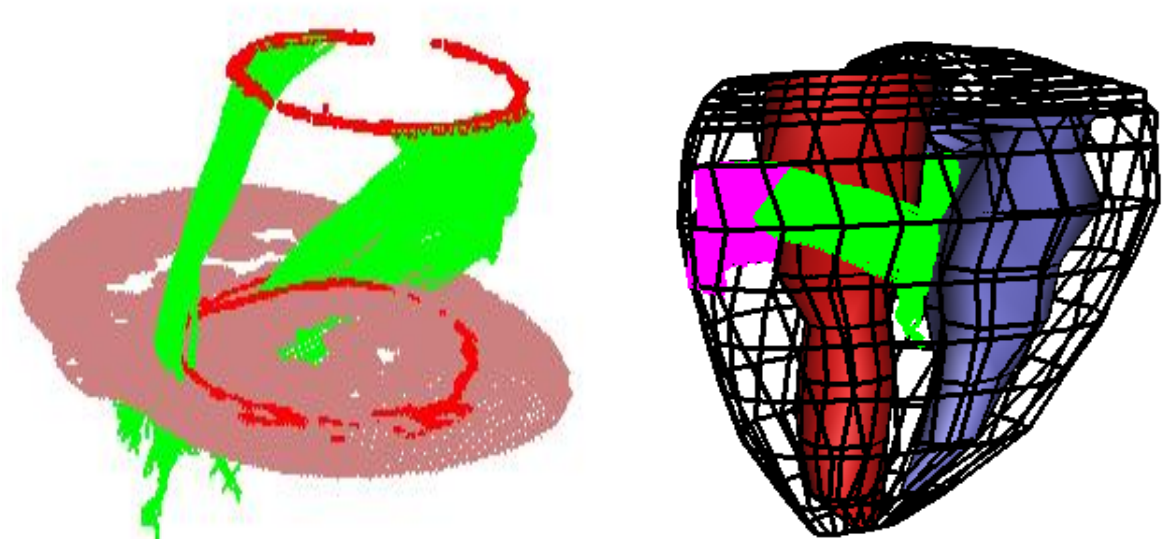

Figure 1. Left: A surface of myocardial fibres in the left ventricle crosses the circles of isovalues of Clairaut's constant. Right: The position of the same surface in the ventricular wall. The anatomical model has been extracted from experimental data.

In Section 2, we turn to the mechanical aspect of the heart modelling and we focus on the derivation of a constitutive law. Note that Peskin was the first who addressed the important issue of understanding from first principles in mechanics why fibres should actually be geodesics, or approximate geodesics, [Peskin, 1989]. Several constitutive laws can be found in the literature, see, for instance, [Bestel et al., 2001], [Cai, 1998], [Fung, 1990], [Nash and Hunter, 2000], [Taber and Perucchio, 2000], [Usyk et al., 2000]. Most of them postulate that the intra-myocardial stress tensor $\sigma$ can be obtained by adding the anisotropic stress tensor due to the cardiac fibres and the stress tensor of the medium in which the fibres bathe.

We choose a different approach and we propose to use a discrete homogenization method, [Tollenaere and Caillerie, 1998]. Let us mention that classical homogenization techniques of continuous media were used with specific application to the myocardium in [Briane, 1993].

\section{Geometrical modelling}

Several studies attempted along the years to describe the anatomical organization of myocardial fibres. We refer to [Streeter, 1979] and [Jouk et al., 2000] for an extensive bibliography. Among other features, it has been noticed that the fibre direction varies gradually from epicardium to endocardium. From their observations, Streeter and his colleagues stated the hypothesis that, myocardial fibres run as geodesics, i.e., paths of shortest length, on a set of nested surfaces. They checked this property on the equatorial part of the left ventricle, [Streeter et al., 1978]. A first part of our work is devoted to try and extend Streeter's results on the whole of the myocardium.

To this aim, we use data obtained by mean of the recently developed polarized light microscopy technique [Jouk et al., 1995]. This technique allows to determine the fibre direction in any material point of the myocardium by measuring two angles.

The particularity of the left ventricle is that its shape is close to a truncated ellipsoid. In particular, it is a body of revolution. This allows to take advantage of a useful invariance property of geodesics of surfaces of revolution, namely the fact that the Clairaut number remains constant along a geodesic curve. We recall that if $r$ denotes the distance from a point of a geodesic to the axis of revolution and if $\theta$ is the angle that the geodesic makes with the parallel at this point, then the Clairaut number is defined by $C=r \cos \theta$. By using Clairaut's 

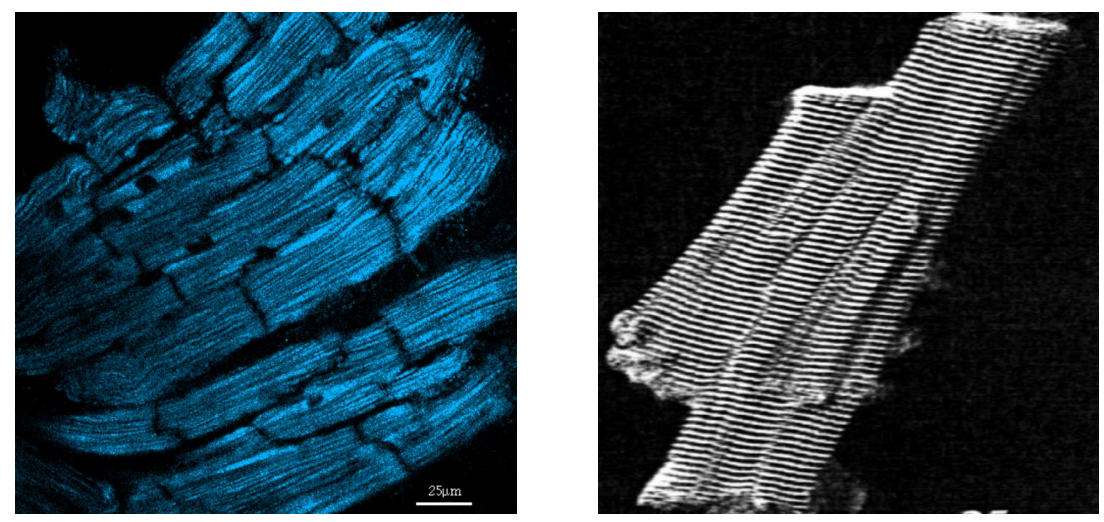

Figure 2. Myocardial cells joined by anastomoses (left) where a principal fibre direction can be determined (right).

property on the experimental data, we were able to validate several consequences of Streeter's hypothesis on the whole of the left ventricle, [Mourad et al., 2001].

More specifically, we computed the quantity $C$ at any point of the left ventricle and plotted its isovalues on horizontal and vertical plane sections. It turns out that the isovalues on a given horizontal section are concentric circles, and, that two concentric circles correspond to a given value of $C$. This observation matches with the hypothesis that epicardial fibres invaginate at the apex where they become endocardial and that they are closed curves deduced one from each other by rotation. And that the same pattern is true for inner fibres. The isovalues of $C$ on vertical sections are nested crescent-shaped meridian curves which agrees with the statement that the fibres run on nested toroidal surfaces, the value $C$ being constant per surface.

In order to refine the global findings obtained up to this point and get an information on each separated fibre, an algorithm allowing to follow fibres point by point and reveal their trajectories has been developed. It can be seen that a given fibre stays on a single surface of isovalue of $C$, which actually proves that along any fibre the quantity $C$ is constant. For a given value $C_{0}$ of Clairaut's constant, we have plotted all fibres starting from one of the two circles corresponding to $C_{0}$ in a given horizontal section. It has been found that, the trace, on different horizontal sections, of the surface formed by these fibres coincides with the circles of isovalue $C_{0}$, see Figure 1. The surfaces show the toroidal structure of the left ventricle and the helicoidal pattern of the fibres.

\section{Mechanical Modelling}

The deformation of the myocardium during its cycle is obtained via the deformation of the myocardial cells, also called myocytes. Our purpose is to derive a constitutive law for the myocardium from the myocytes geometrical organization and from their mechanical behaviour. The myocytes are cylindrical, 60-100 $\mu \mathrm{m}$ long with a 10-20 $\mu \mathrm{m}$ diameter. They are joined by anastomoses into a network where a principal direction can be locally determined. This is the fibre direction, see Figure 2. Because of the smallness of the myocytes with respect to the whole of the myocardium, we can use the discrete homogenization technique originally introduced in [Tollenaere and Caillerie, 1998] and [Moreau and Caillerie, 1998] for quasiperiodic lattice structures in civil engineering. This technique is an adaptation to discrete media of the asymptotic expansion method in the homogenization theory of periodic media.

We choose to model the myocytes arrangement by a system of elastic bars linked by their ends and having a preferred direction, see Figure 3. We assume that external sollicitations are exerted on nodes. As we have to deal 


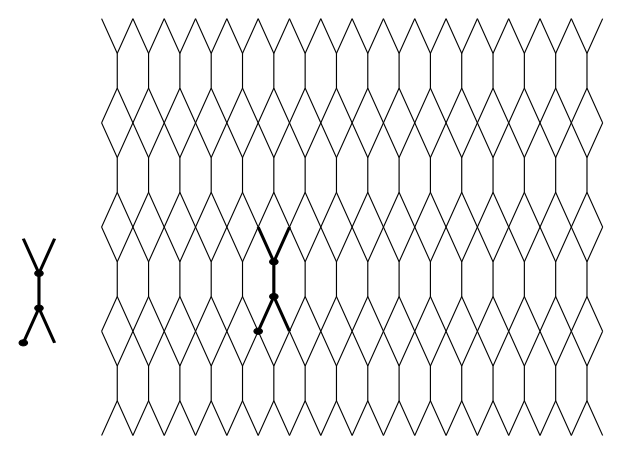

Figure 3. A periodic structure. In bold an elementary period of three nodes and five bars. The elementary period can be much more sophisticated than in this simple example.

with large deformations, we assume that the traction-compression constitutive law of each bar, which expresses the normal tension in the bar as a function of its length, is nonlinearly elastic. Let us mention that we introduce additionally the length at rest of myocytes as a parameter in the constitutive law, see Equation (4). This will allow us to model the activation mechanism. Moreover, in order to take into account the elastic behaviour under sollicitations which are transverse to the myocytes directions, we need to introduce the moments between bars. These moments model the behaviour between myocytes at the anastomoses. We assume that the constitutive law for moments is such that a moment between two bars is a function of their directions, see Equation (5).

Let $\tilde{\mathcal{N}}$ be the set of all nodes $\tilde{n}$ and $\tilde{\mathcal{B}}$ be the set of all bars $\tilde{b}$. Each bar $\tilde{b}$ links two nodes and is oriented. One of the two nodes is the origin and is denoted by $O(\tilde{b})$. The other node is the end and is denoted by $E(\tilde{b})$. Let $\tilde{\mathcal{C}}$ be the set of pairs $\tilde{c}=\left(\tilde{b}, \tilde{b^{\prime}}\right)$ of connected bars. Each pair $\tilde{c}$ is oriented, one of the two bars is the origin and is denoted by $P(\tilde{c})$, the other is the end and is denoted by $D(\tilde{c})$.

External loads $\vec{f}^{e} / \tilde{n}$ are applied on the nodes $\tilde{n}$. They are balanced by internal stresses which are represented in our lattice model by the tension $\vec{T}^{\tilde{b}}$ in bars $\tilde{b}$ and the moments $\vec{M}^{\tilde{c}}$ exerted by each bar $D(\tilde{c})$ on the bar $P(\tilde{c})$. Neglecting the acceleration, the virtual power formulation of balance equations is:

$$
\begin{aligned}
\forall \vec{v}(\tilde{n}), \quad \sum_{\tilde{b} \in \tilde{\mathcal{B}}} \vec{T}^{\tilde{b}} \cdot(\vec{v}(O(\tilde{b}))-\vec{v}(E(\tilde{b})))+\sum_{\tilde{n} \in \tilde{\mathcal{N}}} \vec{f}^{e} / \tilde{n} \cdot \vec{v}(\tilde{n}) & =0, \\
\forall \vec{w}(\tilde{b}), \quad \sum_{\tilde{c} \in \tilde{\mathcal{C}}} \vec{M}^{\tilde{c}} \cdot(\vec{w}(P(\tilde{c}))-\vec{w}(D(\tilde{c})))+\sum_{\tilde{b} \in \tilde{\mathcal{B}}} l^{\tilde{b}}\left(\vec{e}^{\tilde{b}} \wedge \vec{T}^{\tilde{b}}\right) \cdot \vec{w}(\tilde{b}) & =0,
\end{aligned}
$$

where $l^{\tilde{b}}$ is the length of the bar $\tilde{b}$ and $\tilde{e}^{\tilde{b}}$ is its unit vector in the deformed configuration.

Each tension $\vec{T}^{\tilde{b}}$ has two components, one along the bar direction $N^{\tilde{b}} \tilde{e}$ and the other one in the transverse plane $\vec{T}_{t}^{\tilde{b}}$. In other words,

$$
\vec{T}^{\tilde{b}}=N^{\tilde{b}} \bar{e}^{\tilde{b}}+\vec{T}_{t}^{\tilde{b}}
$$

As explained earlier, the constitutive equations for the normal tension $N^{\tilde{b}}$ and the moment $\vec{M}^{\tilde{c}}$ are:

$$
\begin{array}{r}
N^{\tilde{b}}=\mathcal{N}^{\tilde{b}}\left(l^{\tilde{b}}, l_{0}^{\tilde{b}}\right), \\
\vec{M}^{\tilde{c}}=\overrightarrow{\mathcal{M}}^{\tilde{c}}\left(\vec{e}^{P(\tilde{c})}, \vec{e}^{D(\tilde{c})}\right),
\end{array}
$$

where $l_{0}^{\tilde{b}}$ denotes the length of $\tilde{b}$ at rest. 
The unknowns in system $(1),(2),(4),(5)$ are the positions $\vec{R}(\tilde{n})$ of the nodes in the deformed configuration.

Applying the discrete homogenization method consists in assuming that the unknowns $\vec{R}(\tilde{n})$ admit an asymptotic expansion in terms of a small parameter $\varepsilon$ which represents the ratio of the size of an elementary cell over the lattice size. For this prospect, it is convenient to introduce a numbering of nodes and bars which reflects the lattice periodicity. A numbering of cells is given by triplets of integers $\left(\nu^{1}, \nu^{2}, \nu^{3}\right)$. Nodes and bars are then referenced by quadriplets $\left(n, \nu^{1}, \nu^{2}, \nu^{3}\right)$, or $\left(b, \nu^{1}, \nu^{2}, \nu^{3}\right)$, where $n \in \mathcal{N}_{\mathcal{R}}$ and $b \in \mathcal{B}_{\mathcal{R}}$ number the nodes and beams of a reference cell. The assumed expansion reads:

$$
\vec{R}(\tilde{n})=\vec{R}^{0}\left(\lambda^{1 \epsilon}, \lambda^{2 \epsilon}, \lambda^{3 \epsilon}\right)+\epsilon \vec{R}^{n 1}\left(\lambda^{1 \epsilon}, \lambda^{2 \epsilon}, \lambda^{3 \epsilon}\right)+\ldots, \quad \lambda^{i \epsilon}=\epsilon \nu^{i} .
$$

The goal is to identify a limit problem for the macroscopic function $\vec{R}^{0}\left(\lambda^{1}, \lambda^{2}, \lambda^{3}\right)$ where $\lambda^{1}, \lambda^{2}, \lambda^{3}$ are macroscopic curvilinear coordinates ranging over the equivalent continuum space $\omega$.

The response function in (4) and (5) can have several behaviours in terms of $\varepsilon$. Depending on their respective orders of magnitude, several limit models can be obtained. Experiments show that in general, the stiffness of the moments is lower than the traction stiffness. In this study, the ratio of stiffness is taken proportional to $\varepsilon$. This choice actually seems to give the complete macroscopic behaviour in the sense that both effects of traction and moments are present.

Expansion (6) induces expansions for the bar lengths and for their unit vectors. Notice that in this process Taylor expansions are used. As a consequence, the gradient of $\vec{R}^{0}$ appears in the series coefficients. All obtained expressions are then used to expand constitutive and balance equations. All leading terms are denoted by a 0 superscript. This procedure gives rise to the limit macroscopic balance equation which reads

$$
\forall \vec{v}^{0}, \quad-\int_{\omega}\left(\vec{S}^{i 0}+\vec{T}_{t}^{i 0}\right) \cdot \frac{\partial \vec{v}^{0}}{\partial \lambda^{i}} d \lambda+\int_{\omega} \vec{f} \cdot \vec{v}^{0} d \lambda=0,
$$

where,

$$
\vec{S}^{i 0}=\sum_{b \in \mathcal{B}_{\mathcal{R}}} N^{b 0} \vec{e}^{b 0} \delta^{i b}, \vec{T}_{t}^{i 0}=\sum_{b \in \mathcal{B}_{\mathcal{R}}} \vec{T}_{t}^{b 0} \delta^{i b}, \text { and } \vec{f}=\sum_{n \in \mathcal{N}_{\mathcal{R}}} \vec{f}^{e / n}
$$

In the above expressions, $\delta^{i b}=0,-1,1$ according to whether $E(\tilde{b})$ belongs to the same period as $O(\tilde{b})$, or to a previous period, or to the next one in the $\lambda^{i}$ direction.

We have to supplement equation (7) with a macroscopic constitutive law. As is classical in the homogenization of continuous media, the relationship between a macroscopic deformation and macroscopic stresses is obtained through the resolution of a problem set on an elementary cell. This microscopic problem consists of balance equations

$$
\begin{aligned}
\forall \vec{v}^{n}, \sum_{b \in \mathcal{B}_{\mathcal{R}}}\left(N^{b 0} \vec{e}^{b 0}+\vec{T}_{t}^{b 0}\right) \cdot\left(\vec{v}^{O_{R}(b)}-\vec{v}^{E_{R}(b)}\right) & =0, \\
\forall \vec{w}^{b}, \quad \sum_{c \in \mathcal{C}_{\mathcal{R}}} \vec{M}^{c 0} \cdot\left(\vec{w}^{P_{R}(c)}-\vec{w}^{D_{R}(c)}\right)+\sum_{b \in \mathcal{B}_{\mathcal{R}}} l^{b 0}\left(\vec{e}^{b 0} \wedge \vec{T}_{t}^{b 0}\right) \cdot \vec{w}^{b} & =0
\end{aligned}
$$

and of constitutive laws which determine $N^{b 0}, \vec{M}^{c 0}$ in terms of $\vec{R}^{n 1}$ and of $\frac{\partial \vec{R}^{0}}{\partial \lambda^{i}}$. Therefore, for a given $\frac{\partial \vec{R}^{0}}{\partial \lambda^{i}}$, system (9)-(10) and microscopic constitutive laws provide $\vec{R}^{n 1}, N^{b 0}, \vec{M}^{c 0}$ and $\vec{T}_{t}^{b 0}$. Then, equations (8) give $\vec{S}^{i 0}, \vec{T}_{t}^{i 0}$ in terms of $\frac{\partial \vec{R}^{0}}{\partial \lambda^{i}}$. This is the macroscopic constitutive law. 
Our simplified framework does not take into account the extracellular matrix. Work into progress is devoted to add an incompressibilty constraint to the model.

We wish to thank Pierre-Simon Jouk, Yves Usson and Gabrielle Michalowicz who originated this work, provided the experimental data, and shared with us some of their biological knowledge during many fruitful exchanges. We thank Luc Biard and Nicolas Szafran for their help. This work was supported by the Fonds National de la Science (ACI Modélisation mathématique, mécanique et numérique du myocarde) and by the Région Rhône-Alpes (Projet Mathématiques pour ADéMo).

\section{REFERENCES}

[Bestel et al., 2001] Bestel J., Clément F., and Sorine M. (2001) - A biomechanical model of muscle contraction in Medical Image Computing and Computer-Assisted Intervention, Utrecht.

[Briane, 1993] Briane M. (1993) - Three models of non periodic fibrous materials obtained by homogenization, Mathematical Modelling and Numerical Analysis, Vol. 27, pp. 759-775.

[Cai, 1998] Cai H. (1998) - Loi de comportement en grandes déformations du muscle à fibres actives. Application à la mécanique du cœur humain et à sa croissance, Thèse de l'Université de Savoie.

[Fung, 1990] Fung Y.C. (1990) - Biomechanics : mechanical properties of tissues, Springer Verlag, second edition.

[Jouk et al., 1995] Jouk P.S., Usson Y., Michalowicz G., and Parazza F. (1995) - Mapping of the orientation of myocardial cells by means of polarized light and confocal scanning laser microscopy, Microsc. Res. Tech., Vol. 30, pp. 480-490.

[Jouk et al., 2000] Jouk P.S., Usson Y., Michalowicz G., and Grossi L. (2000) - Three-dimensional cartography of the pattern of the myofibres in the second trimester fetal human heart, Anat. Embryol., Vol. 202, pp. 103-118.

[Moreau and Caillerie, 1998] Moreau G. and Caillerie D. (1998) - Continuum modeling of lattice structures in large displacement applications to buckling analysis, Computers and Structures, Vol. 68, pp. 181-189.

[Mourad et al., 2001] Mourad A., Biard L., Caillerie D., Jouk P-S., Raoult A., Szafran N., and Usson Y. (2001) - Geometrical modelling of the fibre organization in the human left ventricle, in Functional Imaging and Modeling of the Heart, Katila, Magnin, Clarysse, Montagnat, Nenonen Eds, LNCS 2230, 32-38, Springer, 2001.

[Nash and Hunter, 2000] Nash M.P. and Hunter P.J. (2000) - Computational mechanics of the heart, Journal of Elasticity, Vol. 61, pp. 113-141.

[Peskin, 1989] Peskin C.S. (1989) - Fiber architecture of the left ventricular wall: An asymptotic analysis, Comm. Pure Appl. Math., Vol XLII, pp. 79-113.

[Ohayon et al., 1998] Ohayon J., Usson Y., Jouk P.S., and Cai H. (1998) - Fibre orientation in human fetal heart and ventricular mechanics : A small perturbation analysis, Comput. Methods Biomechanics Biomedic. Eng., Vol. 2, pp. 83-105.

[Streeter et al., 1978] Streeter D.D., Powers W.E., Ross M.A. and Torrent-Guasp F. (1978) - Three-dimensional fiber orientation in the mammalian left ventricular wall, Cardiovascular System Dynamics, eds., Baan J., Noodergraaf A. and Raines J., M.I.T. Press, Cambridge, Mass, pp. 73-84.

[Streeter, 1979] Streeter D.D. (1979) - Gross morphology and fiber geometry of the heart, in Handbook of Physiology. Thecardiovascular system, Berne R.M., Sperelakis N., Geiger S.R. eds, Am. Phys. Soc., Williams \& Wilkins, Baltimore.

[Taber and Perucchio, 2000] Taber L.A. and Perucchio R. (2000) - Modeling heart development, Journal of Elasticity, Vol. 61, pp. 165-197.

[Tollenaere and Caillerie, 1998] Tollenaere H. and Caillerie D. (1998) - Continuous modeling of lattice structures by homogenization, Advances in Engineering Software, Vol. 29, No. 7-9, pp. 699-705.

[Usyk et al., 2000] Usyk T.P., Mazhari R, and McCulloch A.D. (2000) - Effect of laminar orthotropic myofiber architecture on regional stress and strain in the canine left ventricle, Journal of Elasticity, Vol. 61, pp. 143-165. 